\title{
Insulin resistance and levels of adipokines in patients with untreated early rheumatoid arthritis
}

\author{
Sara Manrique-Arija ${ }^{1}$ - Inmaculada Ureña ${ }^{1}$ Pedro Valdivielso ${ }^{2}$ José Rioja $^{3}$. \\ Francisco G. Jiménez-Núñez ${ }^{1}$ - María V. Irigoyen ${ }^{1} \cdot$ Antonio Fernández-Nebro ${ }^{1}$
}

Received: 9 May 2015 /Revised: 3 October 2015 / Accepted: 21 October 2015 /Published online: 3 November 2015

(C) The Author(s) 2015. This article is published with open access at Springerlink.com

\begin{abstract}
The aim of this study is to investigate the presence of insulin resistance (IR) in patients with untreated early rheumatoid arthritis (ERA) and its relationship with adipokines, inflammatory cytokines, and treatment. In this prospective study, we enrolled 46 ERA patients with a disease duration of $<1$ year, and 45 sex-, age-, race-, and body mass index (BMI)-matched controls. Patients and controls with diabetes or a history of glucocorticoid (GC) or disease-modifying antirheumatic drugs (DMARDs) use were excluded. Patients were assessed at the time of diagnosis (visit 1) and after 6 months of treatment (visit 2). The main outcomes were homeostatic model assessment of IR (HOMA-IR) and $\beta$-cell function (HOMA- $\beta$ ) and quantitative insulin sensitivity check index (QUICKI). A multivariate regression analysis was performed to analyze IR adjusting according to lipids, body composition, physical activity, nutrition, and inflammatory cytokine and adipokine levels. The baseline HOMA-IR, HOMA- $\beta$, and QUICKI values were similar in both groups. However, patients showed lower levels of physical activity, total cholesterol, and high-density lipoprotein. Moreover, the
\end{abstract}

Antonio Fernández-Nebro

afernandezn@uma.es

1 UGC de Reumatología, Instituto de Investigación Biomédica de Málaga (IBIMA), Hospital Regional Universitario de Málaga, Universidad de Málaga, Málaga, Spain

2 UGC de Medicina Interna, Instituto de Investigación Biomédica de Málaga (IBIMA), Hospital Universitario Virgen de la Victoria, Universidad de Málaga, Málaga, Spain

3 Departamento de Medicina y Dermatología, Instituto de Investigación Biomédica de Málaga (IBIMA), Universidad de Málaga, Málaga, Spain inflammatory cytokines and resistin concentrations were higher in patients than controls. Multivariate analysis indicated that BMI and baseline rheumatoid factor levels were positively associated with HOMA-IR and HOMA- $\beta$, and negatively with QUICKI. After DMARD treatment, patients showed improvements in inflammatory parameters and lipids whereas IR remained stable. Furthermore, adiponectin and resistin concentrations decreased slightly. Our data suggest that IR is not present in ERA patients either at diagnosis or at 6 months after treatment. However, symptom duration and fat mass appear to be related.

Keywords Adipokines $\cdot$ DMARDs $\cdot$ Early rheumatoid arthritis $\cdot$ Insulin resistance

\section{Introduction}

Rheumatoid arthritis (RA) is a chronic inflammatory disease associated with premature mortality and multimorbidity [1-4]. The cardiovascular risk of RA patients is comparable to that of patients with diabetes [1]. Both traditional and nontraditional risk factors contribute to accelerated atherogenesis $[5,6]$, including systemic inflammation, GC use, dyslipidemia, hypertension, age, diabetes, and insulin resistance (IR) $[5,7,8]$. The development of systemic inflammation in RA results in energy metabolism disorders, microvascular dysfunction, disability, and physical inactivity [9].

Adipokines, such as leptin, resistin, and adiponectin, critically link nutritional status, physical activity, metabolism, inflammation, and immunity. Similarly, adipokines modulate, and are modulated, by inflammatory cytokines. However, the complex relationships between atherogenesis, cytokines, and adipokines are not yet fully understood [1]. 
IR is not a disease unto itself, but rather a physiological abnormality that greatly increases the chances of developing diabetes and metabolic syndrome [2]. Although RA patients have a high prevalence of metabolic syndrome and factors related to obesity, dyslipidemia, and metabolism disorders, only few studies have been specifically designed to investigate IR in this population. In addition, most of the studies have been conducted in RA patients with longstanding illness, a severe inflammatory burden, comorbidities, and high intake levels of medications that influence the metabolism, including IR [3-7]. Moreover, the relationships among insulin secretion, lipid profiles, proinflammatory cytokines, and adipokines have not been studied in depth in untreated early RA (ERA) patients.

Although certain evidence states that IR is increased in long-term RA cases, it is not clear whether the development of IR accompanies the onset of symptoms or instead occurs subsequently due to poor disease control in some patients. In the present study, we aimed to determine whether patients with ERA have increased IR, and to analyze the relationships among factors known to affect glucose metabolism at ERA diagnosis (visit 1) and after 6 months of treatment with DMARDs (visit 2).

\section{Materials and methods}

\section{Study design and setting}

This is a prospective longitudinal 6-month observation study, which was conducted in the University Hospital of Malaga, Spain. Patients were assessed when ERA diagnosis was made and before beginning treatment with DMARDs or GC (visit 1) and after 6 months of treatment (visit 2). The study was approved by the medical ethics committee, and all subjects provided written informed consent before enrollment.

\section{Subjects}

\section{Patients}

We enrolled consecutive, untreated ERA patients who were referred to our rheumatology outpatient clinic. The inclusion criteria were as follows: a symptom duration at diagnosis of $<12$ months, age of $\geq 16$ years, presence of active disease (DAS28 $\geq 3.2$ ), and diagnosis of RA according to the American College of Rheumatology/European League Against Rheumatism 2010 classification criteria [8]. The exclusion criteria were the presence of any inflammatory or rheumatic disease other than RA (except for secondary Sjögren's syndrome), diabetes or impaired glucose tolerance (IGT), or any noncontrolled general condition. Subjects with active infections, those who were pregnant or breastfeeding, and those with current or previous use of oral antidiabetic drugs, insulin, GC, or DMARDs were excluded. However, patients who were receiving nonsteroidal anti-inflammatory drugs (NSAIDs) were not excluded.

Subjects who had been taking beta-blockers, angiotensinconverting enzyme inhibitors, angiotensin receptor blockers, or lipid-lowering drugs for at least 3 months were included if the dose was the same for at least 28 days.

\section{Controls}

Control subjects, were pair-matched $1: 1$, by sex, age, race, and body mass index (BMI), were selected among nonrheumatic patients from associated primary care centers, who volunteered for the study. The control subjects were those who did not meet the classification criteria for any inflammatory disease. The inclusion/exclusion criteria for control subjects were identical to those used for the patients.

\section{Outcome measures}

The outcome measures were IR measured by the homeostatic model assessment of IR (HOMA-IR), the homeostatic model assessment of $\beta$-cell function (HOMA- $\beta$ ) [9], and the quantitative insulina sensitivity check index (QUICKI) [10].

\section{Other recorded variables and study protocol}

Subjects were assessed through a standardized clinical interview in visit 1 and visit 2 .

Weight, height, BMI (weight/height2), blood pressure (the mean of two measurements obtained 5 min apart after subjects had rested in a supine position for $10 \mathrm{~min}$ ), waist and hip circumferences, and waist-to-hip ratio (WHR) were all measured [10]. The data recorded included the traditional Framingham risk factors, current and previous treatment details, physical activity level, and body composition as measured by dual energy X-ray absorptiometry (DEXA; GE Lunar Prodigy enCORETM 2006 software).

Also, fat mass index (FMI) and free-fat mass index (FFMI) were measured.

Patients and controls completed self-administered semiquantitative questionnaires about their educational level, diet, and physical activity at work and leisure. To detect IGT, subjects with baseline blood glucose levels of $<126 \mathrm{mg} / \mathrm{dL}$ underwent an oral glucose tolerance test (OGTT) in visit 1 . Patients and controls with IGT in OGTT were excluded. Samples were collected after 12-16 h of fasting between 9:00 and 10:00 AM.

Enzymatic methods were used to measure the levels of total cholesterol (TC), triglycerides, and high-density lipoprotein (HDL) cholesterol. In addition, low-density lipoprotein (LDL) cholesterol concentrations were calculated [11]. The serum insulin levels at baseline and $2 \mathrm{~h}$ after loading were 
measured by an enzyme-amplified chemiluminescent assay $\left(\right.$ Immulite $\left.\mathrm{ONE}^{\circledR}\right)$. The serum levels of high-sensitivity C-reactive protein (hsCRP) were measured by standard nephelometry (normal range, $0-6 \mathrm{mg} / \mathrm{L}$ ). The serum levels of tumor necrosis factor alpha (TNF- $\alpha$ ) were measured using an automated immunoassay analyzer (Immulite ${ }^{\circledR}$, Diagnostic Products Corporation, Los Angeles, CA; normal range, 0-8.1 pg/ $\mathrm{mL}$ ). The serum interleukin (IL)-6 levels were measured by an enzyme chemiluminescent assay (QuantiGlo ${ }^{\circledR}$; normal range, $0-5.84 \mathrm{pg} / \mathrm{mL}$ ). Adiponectin (normal value, mean \pm standard deviation [SD], $11.5 \pm 5.9 \mu \mathrm{g} / \mathrm{mL}$ ), resistin (normal value, median [interquartile range $\{\mathrm{IQR}\}], 7.2[5.4-8.5] \mathrm{ng} / \mathrm{mL})$, and leptin levels were measured with ELISA kits (Mediagnost ${ }^{\mathbb{B}}$; normal values were considered to fall between the 5th and 95th percentiles, after adjusting for sex and BMI, according to the manufacturer's recommendations).

Variables of RA included symptom duration, swollen joint count (in 28 joints), tender joint count (in 28 joints), and patient's assessment of disease activity (measured on a 0 $100 \mathrm{~mm}$ visual analog scale). The disease activity score in 28 joints (DAS28) [12] was calculated, and all patients completed a health assessment questionnaire (HAQ) [13]. The patients were managed according to the Clinical Practice Guideline for the Management of RA in Spain (GUIPCAR 2007) [14] and followed up by a rheumatologist in routine clinical practice.

\section{Definitions}

IR was defined as a HOMA-IR score of $\geq 2.75$ based on the 90th percentile of healthy people in a Spanish cohort [15]. IGT and diabetes were diagnosed in accordance with the recommendations of the American Diabetes Association [16]. Smoking was only considered in active smokers. Hypertension was defined as a blood pressure of $\geq 140 / 90 \mathrm{~mm} \mathrm{Hg}$, or ongoing treatment with antihypertensive medications [17].

Obesity was defined according to BMI [18]. Dyslipidemia (LDL cholesterol $>160 \mathrm{mg} / \mathrm{dl}$, triglycerides $>200 \mathrm{mg} / \mathrm{dl}$, and HDL cholesterol $<40 \mathrm{mg} / \mathrm{dl}$ (men) and $<50 \mathrm{mg} / \mathrm{dl}$ (women)) and metabolic syndrome were defined according to the NCEP ATP III $[19,20]$. The estimated cardiovascular risk of fatal cardiovascular disease within 10 years was calculated using the SCORE model. A cutoff point of SCORE $\geq 5 \%$ was used [21]. The presence of inflammatory activity was defined as a DAS28 score of $\geq 3.2$ [12]. FMI was defined as fat mass $(\mathrm{kg}) /$ height squared $\left(\mathrm{m}^{2}\right)$ and FFMI as fat-free mass $(\mathrm{kg}) /$ height squared $\left(\mathrm{m}^{2}\right)$. The value of fat mass and fat-free mass was obtained by DEXA [22].

\section{Statistical analyses}

The sample size was calculated assuming a prevalence of insulin resistance of $19 \%$ in controls and $51 \%$ in patients with recent onset RA [23] $(\mathrm{RR}=2.7)$ and an $\alpha$ and $\beta$ error of 0.05 and 0.20 , respectively. The estimated initial sample was 44 subjects per group, but the sample size increased further assuming that approximately $10 \%$ of patients may be finally excluded from analysis for not meeting inclusion criteria.

Data are presented as means (SD), medians (IQR), or totals with percentages. The baseline characteristics were compared between groups using the $\chi^{2}$ test, the Student's two-tailed $t$ test (Fisher's exact test when necessary), or the Mann-Whitney $U$ test. Paired comparisons of changes from baseline to 6 months in RA patients were performed with the paired $t$ test or Wilcoxon matched-pairs signed rank test, as appropriate. Changes in the categorical variables were evaluated by McNemar's test.

Bivariate correlations were calculated between quantitative variables. The main significant variables were selected as independent variables for multiple linear regression models (backward stepwise elimination), wherein the HOMA-IR, HOMA- $\beta$, or QUICKI was used as dependent variable. As HOMA-IR, HOMA- $\beta$, or QUICKI are not normally distributed, a Box-Cox transformation was performed before introduce them in the multivariate analysis. The analysis was performed using the Methodological and Statistical Assessment Unit of FIMABIS (Malaga, Spain) using The R Statistical software (version 3.1.1). The other analyses were performed with SPSS 21.0 software (IBM Corp, USA). Two-tailed tests and a $5 \%$ significance level were used in all analyses.

\section{Results}

\section{Baseline characteristics}

The study population comprised 56 Caucasian patients with early arthritis. During follow-up, seven patients were excluded because they had arthritis other than RA, and another three patients were excluded because of previously unknown diabetes mellitus.

None of the subjects had IGT. The final analyzed data set included 46 ERA patients and 45 control subjects.

Table 1 lists the baseline characteristics of ERA patients and controls. Although there were no significant differences between ERA patients and controls in the majority of the baseline variables, ERA patients were more likely to have a smoking history $(p=0.035)$ and lower levels of TC and HDL cholesterol than the control subjects $(p=0.024$ and $p=0.044$, respectively). Despite elevated systemic inflammation, people with RA were not significantly more insulin resistant than controls [HOMA-IR, median $(\mathrm{IQR})=1.0(0.3-2.6)$ in ERA patients vs $0.9(0.4-1.8) ; p=0.515]$. The prevalence of IR was $21.7 \%(95 \% \mathrm{CI}, 12.3$ to 35.6$)$ in ERA patients and $15.6 \%(95 \% \mathrm{CI}, 7.7$ to 28.8$)$ in control subjects, the 
Table 1 Baseline characteristics in patients and controls (visit 1)

\begin{tabular}{|c|c|c|c|}
\hline & ERA patients & Controls & $p$ value \\
\hline Age (years), mean (SD) & $51.6(17.6)$ & $50.7(12.7)$ & 0.787 \\
\hline Gender: female, $n(\%)$ & $35(76.1)$ & $33(73.3)$ & 0.763 \\
\hline Time until diagnosis ERA in months, mean (SD) & $5.9(3.5)$ & - & - \\
\hline Smoking, $n(\%)$ & $12(26.1)$ & $4(8.9)$ & 0.035 \\
\hline Baseline hypertension, $n(\%)^{\mathrm{a}}$ & $15(32.6)$ & $7(15.6)$ & 0.057 \\
\hline \multicolumn{4}{|l|}{ Anthropometric features } \\
\hline BMI $\left(\mathrm{kg} / \mathrm{m}^{2}\right)$, mean (SD) & $26.8(4.6)$ & $27.1(3.5)$ & 0.673 \\
\hline Waist circumference $(\mathrm{cm})$, mean $(\mathrm{SD})$ & $89.7(14.6)$ & $90.0(11.4)$ & 0.909 \\
\hline Hip circumference (cm), mean (SD) & $104.5(13.1)$ & $106.0(6.6)$ & 0.484 \\
\hline Waist-hip ratio, mean (SD) & $0.85(0.09)$ & $0.84(0.09)$ & 0.572 \\
\hline Fat mass index, mean (SD) & $10.49(4.25)$ & $10.52(3.25)$ & 0.830 \\
\hline Fat-free mass index, median (IQR) & $15.48(13.88-16.89)$ & $15.03(13.86-17.30)$ & 0.848 \\
\hline \multicolumn{4}{|l|}{ Metabolic features } \\
\hline Total cholesterol (mg/dl), mean (SD) & $195.5(40.3)$ & $214.8(39.5)$ & 0.024 \\
\hline Triglycerides (mg/dl), mean (SD) & $110.3(60.9)$ & $106.7(45.8)$ & 0.750 \\
\hline LDL cholesterol (mg/dl), mean (SD) & $122.8(33.4)$ & $137.0(34.8)$ & 0.053 \\
\hline HDL cholesterol (mg/dl), mean (SD) & $52.2(14.8)$ & $59.1(16.7)$ & 0.044 \\
\hline TG/HDL ratio, mean (SD) & $1.8(1.2-3.0)$ & $2.0(1.0-2.9)$ & 0.585 \\
\hline $\mathrm{TC} / \mathrm{HDL}$ ratio, mean (SD) & $3.97(1.09)$ & $3.91(1.34)$ & 0.819 \\
\hline Dyslipidemia, $n(\%)$ & $36(78.3)$ & $39(86.7)$ & 0.292 \\
\hline Fasting glucose (mg/dl), mean (SD) & $84.5(9.9)$ & $80.9(13.8)$ & 0.165 \\
\hline 2 h OGTT glucose (mg/dl), mean (SD) & $113.9(35.3)$ & $99.1(30.9)$ & 0.036 \\
\hline Metabolic syndrome, $n(\%)$ NCEP ATP III & $7(15.2)$ & $5(11.1)$ & 0.563 \\
\hline Insulin $(\mu \mathrm{IU} / \mathrm{ml})$, median (IQR) & $4.5(1.5-11.9)$ & $4.2(1.6-8.5)$ & 0.821 \\
\hline HOMA-IR, median (IQR) & $1.0(0.3-2.6)$ & $0.9(0.4-1.8)$ & 0.515 \\
\hline HOMA-IR $\geq 2.75, n(\%)$ & $10(21.7)$ & $7(15.6)$ & 0.592 \\
\hline HOMA- $\beta$-cell, median (IQR) & $16.8(4.3-45.9)$ & $14.3(5.1-35.2)$ & 0.943 \\
\hline QUICKI, mean (SD) & $0.39(0.07)$ & $0.40(0.07)$ & 0.549 \\
\hline QUICKI $\leq 0.33, n(\%)$ & $10(21.7)$ & $8(17.8)$ & 0.793 \\
\hline \multicolumn{4}{|l|}{ Acute phase reactants, cytokines and adipokines } \\
\hline hsCRP (mg/l), median (IQR) & $12.3(7.1-23.2)$ & $1.6(1.1-2.1)$ & $<0.001$ \\
\hline ESR $(\mathrm{mm} / \mathrm{h})$, mean $(\mathrm{SD})$ & $32.9(21.4)$ & $7.2(5.4)$ & $<0.001$ \\
\hline TNF-alpha (pg/ml), median (IQR) & $10.7(8.7-12.8)$ & $6.9(6.1-7.9)$ & $<0.001$ \\
\hline IL-6 (pg/ml), median (IQR) & $20.1(5.2-46.0)$ & $1.2(0.7-1.9)$ & $<0.001$ \\
\hline Adiponectin $(\mu \mathrm{g} / \mathrm{ml})$, mean $(\mathrm{SD})$ & $10.4(6.4)$ & $9.2(5.6)$ & 0.352 \\
\hline Resistin (ng/ml), median (IQR) & $7.0(5.7-10.0)$ & $5.4(4.6-7.9)$ & 0.008 \\
\hline Leptin (ng/ml), mean (SD) & $17.2(17.7)$ & $16.1(10.9)$ & 0.744 \\
\hline
\end{tabular}

$E R A$ early rheumatoid arthritis, $N D$ No data, $B M I$ body mass index, $L D L$ low-density lipoprotein, $H D L$ highdensity lipoprotein, $T G / H D L$ triglycerides/high-density lipoprotein, $T C / H D L$ total cholesterol/high-density lipoprotein, OGTT oral glucose tolerance test, NCEP ATP III National Cholesterol Education Program Adult Treatment Panel III, HOMA IR insulin resistance index, HOMA $\beta$-cell $\beta$-cell function index, QUICKI Quantitative Insulin Sensitivity Check Index, $h s C R P$ high-sensitivity $\mathrm{C}$ reactive protein, $E S R$ erythrocyte sedimentation rate, $T N F$-alpha tumor necrosis factor alpha, $I L-6$ interleukin-6

${ }^{\mathrm{a}} \mathrm{BP} \geq 140 / 90$

difference between which was not significant $(p=0.592)$. There were also no major differences in any carbohydrate metabolism parameters between patients and controls, except for a slightly higher 2-h OGTT result in ERA patients than in control subjects $(p=0.036)$ but the values are within the normal range in both groups. The concentrations of TNF- $\alpha$, IL-6, and resistin were significantly higher in ERA patients than in the control subjects $(p<0.001$ for TNF- $\alpha$ and IL- 6 , and $p=0.008$ for resistin), but there were no differences in adiponectin and leptin concentrations between these groups. 
Educational level, diet, drugs, and physical activity

The control subjects had higher educational level than ERA patients (58.5 vs $24.4 \%$ received a university education, $p=$ 0.014). With regard to diet, although ERA patients ate fish more frequently than control subjects ( 51.2 vs $15.6 \%$ ate fish at least three times weekly, $p=0.004)$, no difference was detected for other foods (i.e., eggs, dairy, fruits, vegetables, meats, sausages, sweets, snacks, or alcohol).

Notably, ERA patients showed a lower baseline recreational physical activity level than control subjects (subjects who had never done exercise: 40 vs $11.6 \%[p=0.002]$; subjects who only occasionally had done exercise: $33.3 \%$ vs. $65.1 \%$ $[p=0.002]$ ).

At baseline, 20 (43.5\%) ERA patients were taking NSAIDs on demand, of which $15(75 \%)$ were being treated with ibuprofen, $3(15 \%)$ with naproxen, and $2(10 \%)$ with diclofenac; 1 (2.2\%) control subject was taking NSAIDs $(p<0.001)$, whereas the remaining patients took acetaminophen with or without an opioid. No differences in the use of other drugs were noted between groups.

Only five patients $(10.9 \%)$ and seven controls $(15.6 \%)$ were taking lipid-lowering drugs, and no significant difference was observed between them $(p=0.509)$. After a reanalysis excluding patients taking lipid-lowering drugs, we did not find changes in the results.

Educational level, diet, and physical activity did not show differences.

\section{Baseline relationship between RA risk factors and insulin sensitivity in ERA patients}

Table 2 shows the manner in which key RA baseline variables correlated with HOMA-IR, HOMA- $\beta$, and QUICKI. A positive correlation was noted between the HOMA-IR score and age, duration of RA symptoms, rheumatoid factor (RF) levels, antibodies to cyclic citrullinated peptide (anti-CCP) levels, TNF- $\alpha$ levels, CT/HDL ratio, and parameters related to adiposity. HOMA- $\beta$ score showed essentially the same associations as the HOMA-IR score. QUICKI showed an inverse association with the same variables that correlated with the HOMA-IR score.

The resistin level was positively associated with the IL-6 level $(\rho=0.376 ; p=0.022)$, but negatively associated with TC $(\rho=-0.362 ; p=0.013)$ and HDL cholesterol $(\rho=0.380 ; p=$ 0.012 ) levels. Furthermore, the leptin level showed a positive correlation with weight, BMI $(r=0.717 ; p<0.001)$, total mass $(r=0.480 ; p=0.001)$, total fat $(r=0.795 ; p<0.001)$, and waist $(r=0.463 ; p=0.001)$ and hip $(r=0.636 ; p<0.001)$ circumferences. Total adiponectin level shows no correlation with any of the above variables.

Furthermore, the TNF- $\alpha$, but not IL-6 level, was positively correlated with BMI $(\rho=0.364 ; p=0.013)$, waist $(\rho=0.342$; $p=0.020)$ circumference, WHR $(\rho=0.392 ; p=0.007)$, triglycerides/HDL ratio $(\rho=0.388 ; p=0.010)$, TC/HDL ratio ( $\rho=0.415 ; p=0.006)$, and triglyceride levels $(\rho=0.335 ; p=$ $0.023)$, fasting glucose levels $(\rho=0.313 ; p=0.034)$, and fasting insulin levels ( $\rho=0.291 ; p=0.049)$. No correlation between TNF- $\alpha$ level and DAS28 score was noted.

Table 3 shows the multivariate linear regression models for HOMA-IR, HOMA- $\beta$, and QUICKI. According to these analysis models, both total fat and symptom duration were the only predictors of the HOMA-IR, HOMA- $\beta$, and QUICKI scores.

\section{Six-month follow-up examination in ERA patients}

Table 4 shows the characteristics of ERA patients at baseline and after 6 months.

Approximately $76 \%$ of the cases were RF-positive and $72 \%$ were anti-CCP-positive.

No patients had extra-articular disease manifestations or a history of GC or DMARD use. All patients exhibited disease activity. One patient was lost to follow-up. After the baseline evaluation, ERA patients received treatment according to their rheumatologist's criteria (Table 4). All ERA patients received escalated dosages of methotrexate, and $36(56.5 \%)$ of these patients received a tapering regimen of concomitant GC (median [IQR] initial dose, 10 [5-20] mg/day). Patients treated with GC had a higher DAS28 score at baseline (mean [SD] 6.0 [1.3] vs 4.7 [0.9]; $p=0.001)$ and worse HAQ score (1.519 [0.822] vs 0.984 [0.456]). We did not find any correlation between the GC and the level of insulin resistance measured by HOMA -RI (Spearman's rho 0.123; $p=0.406$ ). During the 6-month follow-up, one patient switched to sulfasalazine, and two patients received combined treatment. At 6 months, 40 patients $(86.9 \%)$ were treated with NSAIDs, $25(62.5 \%)$ with ibuprofen, $9(22.5 \%)$ with naproxen, $4(10 \%)$ with indomethacin, and 2 (5\%) with diclofenac. Most patients (32; $80 \%)$ were taking NSAIDs on demand. After 6 months of treatment, approximately $70 \%$ of the ERA patients had a low DAS28 score. In addition, there was a slight improvement of physical activity (patients who do exercise: $32.6 \%$ at baseline vs $56.5 \%$ after 6 months; McNemar's test, $p=0.091$ ), which was closer to the levels of physical activity noted in the controls $(65.1 \%)$. There were no significant differences between the anthropometric variables, blood pressure, SCORE risk and metabolic characteristics, HOMA-IR, HOMA- $\beta$, QUICKI, fasting glucose, and fasting insulin levels at baseline and at 6-month evaluation (Table 4). However, after 6 months of treatment, we observed an improvement in lipid profiles, whereas the median concentrations of cytokines and adipokines changed. Adiponectin, resistin, TNF- $\alpha$, and especially IL-6 levels showed a marked decline. In contrast, leptin concentrations were slightly, but not significantly, increased. 
Table 2 Bivariate correlations of patient characteristics with HOMA-IR, HOMA-B, and QUICKI

\begin{tabular}{|c|c|c|c|}
\hline VARIABLE & $\begin{array}{l}\text { HOMA-IR } \\
\text { Spearman } \rho\end{array}$ & $\begin{array}{l}\text { HOMA-B } \\
\text { Spearman } \rho\end{array}$ & $\begin{array}{l}\text { QUICKI } \\
\text { Spearman } \rho / r\end{array}$ \\
\hline Age (years) & $0.422 * *$ & $0.431 *$ & -0.417 \\
\hline Duration of symptoms (months) & $0.370^{*}$ & $0.368^{*}$ & $-0.358^{*}$ \\
\hline Rheumatoid factor (IU) & $0.380 *$ & $0.390^{*}$ & $-0.380^{*}$ \\
\hline Anti-CCP antibodies (IU) & $0.308^{*}$ & $0.252^{*}$ & $-0.308^{*}$ \\
\hline DAS28 & 0.014 & 0.056 & -0.085 \\
\hline hsCRP (mg/L) & 0.055 & 0.068 & -0.055 \\
\hline $\operatorname{ESR}(\mathrm{mm} / \mathrm{h})$ & 0.035 & 0.049 & -0.095 \\
\hline BMI $\left(\mathrm{kg} / \mathrm{m}^{2}\right)$ & $0.449 * *$ & $0.456^{* *}$ & $-0.443 * *$ \\
\hline Waist circumference $(\mathrm{cm})$ & $0.483 * *$ & $0.476^{* *}$ & $-0.450 * *$ \\
\hline Hip circumference $(\mathrm{cm})$ & $0.432 * *$ & $0.429 * *$ & $-0.329 *$ \\
\hline Waist-hip ratio & $0.376^{*}$ & $0.349^{*}$ & $-0.322 *$ \\
\hline Total fat $(\mathrm{kg})$ & $0.555 * * *$ & $0.564 * * *$ & $-0.487 * *$ \\
\hline Total free-fat $(\mathrm{kg})$ & 0.205 & 0.183 & -0.029 \\
\hline Total mass (kg) & $0.430^{*}$ & $0.400 * *$ & -0.353 \\
\hline Systolic blood pressure ( $\mathrm{mm} \mathrm{Hg}$ ) & 0.141 & 0.149 & -0.256 \\
\hline Total cholesterol (mg/dl) & 0.177 & 0.206 & -0.198 \\
\hline LDL cholesterol (mg/dl) & 0.198 & 0.211 & -0.239 \\
\hline HDL cholesterol (mg/dl) & -0.193 & -0.221 & 0.263 \\
\hline Triglycerides (mg/dl) & 0.152 & 0.232 & -0.272 \\
\hline TG/HDL ratio & 0.243 & $0.317^{*}$ & -0.243 \\
\hline $\mathrm{CT} / \mathrm{HDL}$ ratio & 0.317 & $0.356^{*}$ & $-0.380^{*}$ \\
\hline Adiponectin $(\mu \mathrm{g} / \mathrm{ml})$ & -0.095 & -0.044 & -0.010 \\
\hline Resistin (ng/ml) & 0.139 & 0.197 & -0.139 \\
\hline Leptin (ng/ml) & 0.253 & 0.279 & -0.286 \\
\hline TNF-alpha (pg/ml) & $0.292 *$ & $0.284 *$ & $-0.292^{*}$ \\
\hline IL-6 (pg/ml) & -0.002 & 0.050 & -0.093 \\
\hline
\end{tabular}

HOMA IR insulin resistance index, HOMA $\beta$-cell $\beta$-cell function index, QUICKI, Quantitative Insulin Sensitivity Check Index, Anti-CCP anti-cyclic citrullinated peptide, DAS28 Disease Activity Score in 28 Joints, $h s C R P$ highsensitivity $\mathrm{C}$ reactive protein, ESR erythrocyte sedimentation rate, $B M I$ body mass index, $L D L$ low-density lipoprotein, $H D L$ high-density lipoprotein, $T G / H D L$ triglycerides/high density lipoprotein, $T C / H D L$ total cholesterol/high-density lipoprotein, TNF-alpha tumor necrosis factor alpha, IL-6 interleukin-6

${ }^{*} p<0.05 ; * * p>0.005 ; * * * p<0.001$
None of the parameters mentioned above were affected by GC treatment after 6 months, with the exception of larger reductions in IL-6 (mean [SD] -38.5 [45.8] vs -11.3 [19.2] $\mathrm{pg} / \mathrm{mL} ; p=0.028)$, hsCRP $(-21.2[31.8]$ vs $-1.6[9.1] \mathrm{mg} / \mathrm{L}$; $p=0.008)$, and ESR $(-17.4[20.2]$ vs $-6.3[11.7] \mathrm{mm} / \mathrm{h} ; p=$ 0.049), as compared to ERA patients who did not take GC.

\section{Discussion}

We noted that there were no differences in the HOMA-IR or HOMA- $\beta$ scores, or QUICKI between ERA patients and matched control subjects.

Several studies have shown that RA patients have impaired fasting insulin sensitivity [3, 7, 23-25]. However, most of these studies were either conducted in patients with longstanding, treated RA or involved non-BMI-matched controls. Therefore, it is not clear whether this impaired insulin sensitivity was present from the time of RA development or if it developed over time in association with lifestyle changes, persistent inflammation, drug exposure, and other factors.

To our knowledge, few studies have investigated the presence of IR in ERA [25-27].

One of these was a noncontrolled study that included 196 patients treated with DMARDs and/or GC [26]; therefore, their results cannot be compared with our findings. The other study included 66 untreated RA patients with a mean disease duration of 9.8 months and 40 sex- and age-matched controls [25]; this study indicated that HOMA-IR score was higher in ERA patients than in the control subjects. However, these results may differ from the findings of the current study because of several important methodological reasons, such as 
Table 3 Multivariate linear regression models for HOMA-IR (Box-Cox), HOMA-B (Box-Cox), and QUICKI (Box-Cox) in ERA patients

\begin{tabular}{|c|c|c|c|c|}
\hline Predictor & $\mathrm{B}$ & $95 \% \mathrm{CI}$ for $\mathrm{B}$ & $p$ value & $R^{2}$ \\
\hline Dependent variable: HOMA-IR ${ }^{\mathrm{a}, \mathrm{b}}$ & & & & 0.409 \\
\hline Total fat mass (kg) & 0.00005 & 0.00002 to 0.00009 & 0.001 & \\
\hline Symptom duration (months) & 0.105123 & 0.009682 to 0.200564 & 0.032 & \\
\hline $\begin{array}{l}\text { Dependent variable: } \\
\text { HOMA-B }^{\mathrm{b}, \mathrm{c}}\end{array}$ & & & & 0.371 \\
\hline Total fat mass (kg) & 0.000047 & 0.000016 to 0.000077 & 0.004 & \\
\hline Duration of symptoms (months) & 0.096256 & 0.003768 to 0.188745 & 0.042 & \\
\hline Dependent variable: $\mathrm{QUICKI}^{\mathrm{c}}$ & & & & 0.413 \\
\hline Total fat mass $(\mathrm{kg})$ & -0.000008 & -0.000013 to -0.000004 & 0.001 & \\
\hline Duration of symptoms (months) & -0.015484 & -0.029685 to -0.001284 & 0.034 & \\
\hline
\end{tabular}

${ }^{\mathrm{a}}$ Independent variables: age, total fat, waist circumference, duration of symptoms (from onset of symptoms to RA-diagnosis), TNF $\alpha$, RF, and TC/HDL ratio

${ }^{\mathrm{b}}$ Box-Cox transformed: Lambda values used for HOMA-IR, HOMA-B, and QUICKI, were $-0.151,-0.104$, and 0.125 , respectively

${ }^{\mathrm{c}}$ Independent variables: age, total fat, waist circumference, duration of symptoms (from onset of symptoms to RA-diagnosis), TNF $\alpha, \mathrm{RF}, \mathrm{TC} / \mathrm{HDL}$ ratio, and $\mathrm{TG} / \mathrm{HDL}$ ratio

the presence of fewer control subjects than cases, a longer disease duration (9.8 vs 5.9 months in our study), a significantly higher BMI in ERA patients than in control subjects, and the lack of investigation of impaired glucose tolerance in the previous study; in addition, fat mass and body fat distribution were not evaluated. A sedentary lifestyle, overweight/ obesity, central adiposity, and impaired glucose tolerance are widely known to be associated with IR [28]. Furthermore, Sahin et al. did not study the effect of both the evolution time and early treatment of ERA on the IR.

The third one is a cross-sectional study [27]. We also found some differences from our study, the most important being a longer disease duration (9.2 vs 5.9 months in our study). Moreover, they did not study impaired glucose tolerance, other adipokines and cytokines, body composition, diet, or exercise. Furthermore, the effect of the evolution time and early treatment of ERA on the Insulin resistance was not analyzed. The only predictors of IR identified in the present study were total fat and RA symptom duration. These results suggest that IR may not be present during the few first months of arthritis but may develop subsequently if poorly controlled inflammatory disease persists and if there is an increase in the amount of fat mass.

This increase in fat mass might be explained in part by the presence of lower physical activity, which is associated with pain and physical dysfunction caused by arthritis. In fact, the ERA patients in the present study reported a low baseline level of recreational physical activity, which improved when arthritis was controlled. These results are consistent with those found in previous studies $[29,30]$.

There is considerable evidence that supports the association between IR and the presence of increased proinflammatory cytokine levels in longstanding RA [3, 4, 24, 25]. Nevertheless, the only proinflammatory factor that showed some correlation with IR in the present study was TNF- $\alpha$. This cytokine impairs insulin sensitivity and is linked to obesity and diabetes [24]; moreover, it downregulates tyrosine kinase activity and interferes with signaling pathways [31]. According to our results, TNF- $\alpha$ might exert an even greater and faster influence on metabolic processes than IL- 6 because, in addition to its previously reported correlation with IR, it is also correlated with central adiposity and lipid profiles [31].

Besides proinflammatory cytokines, adipokines produced by fat tissue may also have effects on glucose homeostasis, appetite, and inflammatory responses [32, 33].

Consistent with results of previous studies, our results did not show differences in leptin and adiponectin concentrations between ERA patients and control subjects [34, 35]. In fact, leptin concentrations in the ERA patients in the present study were positively correlated with anthropometric variables and IR, but not with inflammatory parameters. In contrast, the resistin concentration was positively correlated with the IL-6 level and HAQ results. All of these results are also consistent with those previously published by other authors [36-38].

After their diagnosis, all patients were treated with methotrexate and $>50 \%$ of these patients received low doses of GC. This treatment yielded a significant improvement of arthritis and physical function in most patients and a decline of acute phase reactants and of resistin, adiponectin, TNF- $\alpha$, and especially IL-6 levels. In our opinion, the improvement in physical function and the recreational physical activity possibly contributed to the maintenance of normal insulin sensitivity after 6 months of treatment. In addition, methotrexate is independently associated with a reduced propensity for metabolic syndrome, thus suggesting a drug-specific mechanism [39].

In contrast, GC use did not influence IR or adipokine levels in our patients after 6 months. This may be due to the complex relationship between GC and IR; in the short term perhaps, the 
Table 4 Characteristics of the ERA patients at baseline (visit 1) and after 6 months (visit 2)

\begin{tabular}{|c|c|c|c|}
\hline Variable & Baseline & 6 months & $\begin{array}{l}p \\
\text { value }\end{array}$ \\
\hline \multicolumn{4}{|l|}{ Arthritis characteristics } \\
\hline Disease duration (months), mean (SD) & $5.9(3.5)$ & - & \\
\hline No. of swollen joints ( 28 assessed) & $8(4-15)$ & $1(0-3)$ & $<0.001$ \\
\hline No. of tender joints (28 assessed) & $4(2-11)$ & $0(0-2)$ & $<0.001$ \\
\hline $\begin{array}{l}\text { Patient's assessment of disease activity (VAS, mm), } \\
\text { mean (SD) }\end{array}$ & $66.3(20.9)$ & $33.9(24.5)$ & $<0.001$ \\
\hline hsCRP (mg/l), median (IQR) & $12.3(7.1-23.2)$ & $5.6(3.0-9.9)$ & $<0.001$ \\
\hline $\operatorname{ESR}(\mathrm{mm} / \mathrm{h})$, mean $(\mathrm{SD})$ & $32.9(21.4)$ & $18.7(12.0)$ & $<0.001$ \\
\hline RF (IU/l), median (IQR) & $43.8(17.9-90.5)$ & $20.2(10.0-80.5)$ & 0.071 \\
\hline Anti-CCP (IU), median (IQR) & $108(3.8-341)$ & $171.0(1.6-340.0)$ & 0.884 \\
\hline DAS28, mean (SD) & $5.5(1.3)$ & $3.2(1.4)$ & $<0.001$ \\
\hline DAS28 <3.2, $n(\%)$ & $0(0)$ & $32(69.6)$ & $<0.001$ \\
\hline DAS28 <2.6, $n(\%)$ & $0(0)$ & $16(34.8)$ & $<0.001$ \\
\hline HAQ, mean (SD) & $1.3(0.7)$ & $0.548(0.567)$ & $<0.001$ \\
\hline \multicolumn{4}{|l|}{ Treatment } \\
\hline NSAIDs, $n(\%)$ & $20(43.5)$ & $40(86.9)$ & $<0.001$ \\
\hline Glucocorticoids, $n(\%)$ & - & $4(9.6)$ & \\
\hline Doses (mg/day), median (IQR) & & $5.0(3.1-10.6)$ & \\
\hline Dose maximum (mg/day), mean (SD) & & $14.4(11.3)$ & \\
\hline Methotrexate, $n(\%)$ & - & $43(93.5)$ & \\
\hline Doses (mg/week), median (IQR) & & $17.5(15.0-20.0)$ & \\
\hline Methotrexate plus Leflunomide, $n(\%)$ & & $1(2.2)$ & \\
\hline Methotrexate plus sulfasalazine, $n(\%)$ & & $1(2.2)$ & \\
\hline Sulfasalazine, $n(\%)$ & - & $1(2.2)$ & \\
\hline \multicolumn{4}{|l|}{ Anthropometric features } \\
\hline BMI, mean (SD) & $26.8(4.7)$ & $27.1(5.3)$ & 0.061 \\
\hline Fat mass index, mean (SD) & $10.49(4.25)$ & $10.63(4.36)$ & 0.107 \\
\hline Fat-free mass index, median (IQR) & $\begin{array}{l}15.48(13.88- \\
16.89)\end{array}$ & $\begin{array}{l}14.85(13.58- \\
16.30)\end{array}$ & 0.411 \\
\hline \multicolumn{4}{|l|}{ Metabolic features } \\
\hline Total cholesterol (mg/dl), mean (SD) & $195.5(40.3)$ & $195.0(38.9)$ & 0.977 \\
\hline Triglycerides (mg/dl), mean (SD) & $110.3(60.9)$ & $116.1(63.8)$ & 0.441 \\
\hline LDL cholesterol (mg/dl), mean (SD) & $122.8(33.4)$ & $118.6(31.2)$ & 0.300 \\
\hline LDL cholesterol >100 mg/dl, $n(\%)$ & $31(67.4)$ & $15(32.6)$ & 0.006 \\
\hline HDL cholesterol (mg/dl), mean (SD) & $52.2(14.8)$ & $56.2(14.6)$ & 0.010 \\
\hline Low HDL cholesterol, $n(\%)$ & $13(28.3)$ & $9(19.6)$ & 0.289 \\
\hline $\mathrm{TG} / \mathrm{HDL}$ ratio, mean (SD) & $1.9(1.2-3.0)$ & $1.6(1.4-2.0)$ & 0.289 \\
\hline $\mathrm{TC} / \mathrm{HDL}$ ratio, mean (SD) & $3.83(1.05)$ & $3.52(0.72)$ & 0.084 \\
\hline Fasting Glucose (mg/dl), mean (SD) & $84.5(9.9)$ & $83.3(10.7)$ & 0.412 \\
\hline Fasting Insulin $(\mu \mathrm{IU} / \mathrm{ml})$, median (IQR) & $4.5(1.5-11.9)$ & $5.0(1.5-10.2)$ & 0.876 \\
\hline HOMA-IR, median (IQR) & $1.0(0.3-2.6)$ & $1.0(0.3-1.9)$ & 0.981 \\
\hline HOMA-IR $\geq 2.75, n(\%)$ & $10(21,7)$ & $8(17.4)$ & 0.754 \\
\hline HOMA- $\beta$-cell, median (IQR) & $16.8(4.3-45.9)$ & $18.5(3.4-47.6)$ & 0.791 \\
\hline QUICKI, mean (SD) & $0.39(0.07)$ & $0.40(0.07)$ & 0.477 \\
\hline QUICKI $\leq 0.33, n(\%)$ & $10(21.7)$ & $8(17.4)$ & 1.000 \\
\hline \multicolumn{4}{|l|}{ Cytokines and adipokines } \\
\hline TNF-alpha (pg/ml), median (IQR) & $10.6(8.7-12.8)$ & $7.8(6.7-9.5)$ & $<0.001$ \\
\hline High TNF-alpha, $n(\%)$ & $37(80.4)$ & $15(32.6)$ & 0.004 \\
\hline IL-6 (pg/ml), median (IQR) & $20.1(5.2-46.0)$ & $2.9(1.4-8.6)$ & $<0.001$ \\
\hline High IL-6, $n(\%)$ & $32(70.3)$ & $15(32.6)$ & $<0.001$ \\
\hline
\end{tabular}


Table 4 (continued)

\begin{tabular}{|c|c|c|c|}
\hline Variable & Baseline & 6 months & $\begin{array}{l}p \\
\text { value }\end{array}$ \\
\hline Adiponectin $(\mu \mathrm{g} / \mathrm{ml})$, mean $(\mathrm{SD})$ & $10.4(6.4)$ & $9.0(5.0)$ & 0.001 \\
\hline Resistin (ng/ml), median (IQR) & $7.0(5.7-10.0)$ & $5.6(4.6-8.8)$ & 0.002 \\
\hline Leptin (ng/ml), mean (SD) & $17.2(17.7)$ & $21.28(23.2)$ & 0.091 \\
\hline \multicolumn{4}{|l|}{ Cardiovascular risk } \\
\hline $\operatorname{SCORE}(\geq 5 \%)$ & $45(97.8)$ & $45(97.8)$ & 1.000 \\
\hline
\end{tabular}

$E R A$ early rheumatoid arthritis, $V A S$ visual analogic scale, $h S C R P$ high-sensitivity $\mathrm{C}$ reactive protein, ESR erythrocyte sedimentation rate, $R F$ rheumatoid factor, Anti-CCP anti-cyclic citrullinated pPeptide, $D A S 28$ Disease Activity Score in 28 Joints, HAQ Health Assessment Questionnaire, NSAIDs nonsteroidal anti-inflammatory drugs, $L D L$ low-density lipoprotein, $H D L$ high-density lipoprotein, $T G / H D L$ triglycerides/high-density lipoprotein, TC/HDL total cholesterol/high-density lipoprotein, HOMA IR insulin resistance index, HOMA $\beta$-cell $\beta$-cell function index, QUICKI Quantitative Insulin Sensitivity Check Index, TNF-alpha Tumor necrosis factor alpha, IL-6 interleukin-6, SCORE Systemic Coronary Risk Evaluation model

potent anti-inflammatory effects of GCs overcome their adverse effects on body composition and glucose metabolism $[40,41]$. We believe that these results reinforce the importance of an early diagnosis and treatment of RA, especially with methotrexate and a short course of low-dose GC, because these could prevent the long-term deleterious consequences of RA on both the joints and metabolism. Therefore, our results do not contradict, but instead complement those observed in patients with longstanding RA.

Thus far, no longitudinal study has investigated the effects of DMARDs on adipokine levels in early treatment-naive RA. Instead, there have only been a few studies in a small number of chronic RA patients [35, 42]. Our results are consistent with those observed after an effective suppression of inflammation during anti-TNF therapy $[43,44]$ but are inconsistent with those observed after several types of DMARD treatment [35, 42]. Nevertheless, these authors do not provide data on the patients' response to DMARD treatment. Six months after treatment initiation, the concentrations of cytokines and adipokines significantly changed in our patients.

Resistin was the only adipokine that was elevated in RA patients, as has also been observed in other studies [36]. With regard to lipid levels, several studies have reported that patients with ERA are characterized by an atherogenic lipid profile that improves after treatment [45]. In the present study, after 6 months of treatment, an improved lipid profile was observed in accordance with earlier studies [45].

Although a larger sample size might have been helpful, the enrollment of ERA patients who were never exposed to DMARDs or GC was an already difficult task.

Moreover, although the use of HOMA instead of the hyperinsulinemic euglycemic clamp method may seem as a limitation, indirect methods such as HOMA and QUICKI have been validated for use. They are reliable indices and clamp substitutes for measuring IR in epidemiological studies, clinical trials, and clinical practice [46].
In conclusion, our data suggested that IR was not present in ERA patients at diagnosis either 6 months after treatment. However, the symptom duration and fat mass appeared to show a direct relationship. Similarly, early treatment of RA with methotrexate might prevent a further increase of IR.

Acknowledgments We are grateful to Olga Pérez (from the FIMABIS) for her statistical advice.

This research was supported by Andalusian Public Health System (BOJA no. 149, 30 July 2007; application number PI 0245/2007) and Andalusian Foundation of Rheumatology grant (2008).

\section{Compliance with ethical standards}

\section{Disclosures None.}

Open Access This article is distributed under the terms of the Creative Commons Attribution 4.0 International License (http:// creativecommons.org/licenses/by/4.0/), which permits unrestricted use, distribution, and reproduction in any medium, provided you give appropriate credit to the original author(s) and the source, provide a link to the Creative Commons license, and indicate if changes were made.

\section{References}

1. Wasko MC, Kay J, Hsia EC, Rahman MU (2011) Diabetes mellitus and insulin resistance in patients with rheumatoid arthritis: risk reduction in a chronic inflammatory disease. Arthritis Care Res (Hoboken) 63:512-521

2. Reaven G (2004) The metabolic syndrome or the insulin resistance syndrome? Different names, different concepts, and different goals. Endocrinol Metab Clin North Am 33:283-303

3. La Montagna G, Cacciapuoti F, Buono R et al (2007) Insulin resistance is an independent risk factor for atherosclerosis in rheumatoid arthritis. Diab Vasc Dis Res 4:130-135

4. Chung CP, Oeser A, Solus JF et al (2008) Inflammation-associated insulin resistance: differential effects in rheumatoid arthritis and systemic lupus erythematosus define potential mechanisms. Arthritis Rheum 58:2105-2112 
5. Dessein PH, Joffe BI (2006) Insulin resistance and impaired beta cell function in rheumatoid arthritis. Arthritis Rheum 54:27652775

6. Dessein PH, Norton GR, Woodiwiss AJ et al (2007) Independent role of conventional cardiovascular risk factors as predictors of Creactive protein concentrations in rheumatoid arthritis. J Rheumatol 34:681-688

7. AbouAssi H, Tune KN, Gilmore B et al (2014) Adipose depots, not disease-related factors, account for skeletal muscle insulin sensitivity in established and treated rheumatoid arthritis. J Rheumatol 41: 1974-1979

8. Aletaha D, Neogi T, Silman AJ et al (2010) Rheumatoid arthritis classification criteria: an American College of Rheumatology/ European League Against Rheumatism collaborative initiative. Arthritis Rheum 62:2569-2581

9. Matthews DR, Hosker JP, Rudenski AS et al (1995) Homeostasis model assessment: insulin resistance and beta-cell function from fasting plasma glucose and insulin concentrations in man. Diabetologia 28:412-419

10. Katz A, Nambi SS, Mather K et al (2000) Quantitative insulin sensitivity check index: a simple, accurate method for assessing insulin sensitivity in humans. J Clin Endocrinol Metab 85:24022410

11. Friedewald WT, Levy RI, Fredrickson DS (1972) Estimation of the concentration of low-density lipoprotein cholesterol in plasma, without use of the preparative ultracentrifuge. Clin Chem 18:499 502

12. Prevoo ML, van 't Hof MA, Kuper HH et al (1995) Modified disease activity scores that include twenty-eight-joint counts. Development and validation in a prospective longitudinal study of patients with rheumatoid arthritis. Arthritis Rheum 38:44-48

13. Esteve-Vives J, Batlle-Gualda E, Reig A (1993) Spanish version of the Health Assessment Questionnaire: reliability, validity and transcultural equivalency. Grupo para la Adaptacian del HAQ a la Población Española. J Rheumatol 20:2116-2122

14. GUIPCAR (2007) Guía de Práctica Clínica para el manejo de la Artritis Reumatoide en España. Sociedad Española de Reumatología. http://www.ser.es/Contenidos_PracticaClinica/ Guias Practica Clinica.html. Accessed 25 June 2012

15. Soriguer F, Gutierrez-Repiso C, Rubio-Martin E et al (2013) Metabolically healthy but obese, a matter of time? Findings from the prospective Pizarra study. J Clin Endocrinol Metab 98:23182325

16. American Diabetes Association (2010) Diagnosis and classification of diabetes mellitus. Diabetes Care 33:S62-S69

17. Mancia G, De Backer G, Dominiczak A et al (2007) ESH/ESC 2007 Guidelines for the management of arterial hypertension. Rev Esp Cardiol 60:968.e1-94

18. WHO (2000) Obesity: preventing and managing the global epidemic. Report of a WHO consultation. World Health Organ Tech Rep Ser 894, 1-253

19. Executive Summary of The Third Report of The National Cholesterol Education Program (NCEP) (2001) Expert panel on detection, evaluation, and treatment of high blood cholesterol in adults (adult treatment panel III). JAMA 285:2486-2497

20. Nathan DM, Buse JB, Davidson MB et al (2006) Management of hyperglycaemia in type 2 diabetes: a consensus algorithm for the initiation and adjustment of therapy. A consensus statement from the American Diabetes Association and the European Association for the Study of Diabetes. Diabetologia 49:1711-1721

21. Conroy RM, Pyorala K, Fitzgerald AP et al (2003) Estimation of ten-year risk of fatal cardiovascular disease in Europe: the SCORE project. Eur Heart J 24:987-1003

22. VanItallie TB, Yang MU, Heymsfield SB et al (1990) Height normalized indices of the body's fat-free mass and fat mass: potentially useful indicators of nutritional status. Am J Clin Nutr 52:953-959

23. Chung CP, Oeser A, Solus JF et al (2008) Prevalence of the metabolic syndrome is increased in rheumatoid arthritis and is associated with coronary atherosclerosis. Atherosclerosis 196:756-763

24. Dessein PH, Joffe BI, Stanwix A et al (2002) The acute phase response does not fully predict the presence of insulin resistance and dyslipidemia in inflammatory arthritis. J Rheumatol 29:462-466

25. Shahin D, Eltoraby E, Mesbah A et al (2010) Insulin resistance in early untreated rheumatoid arthritis patients. Clin Biochem 43:661665

26. Mirjafari H, Farragher TM, Verstappen SM et al (2011) Seropositivity is associated with insulin resistance in patients with early inflammatory polyarthritis: results from the Norfolk Arthritis Register (NOAR): an observational study. Arthritis Res Ther 13: R159

27. El-Hini SH, Mohamed FI, Hassan AA et al (2013) Visfatin and adiponectin as novel markers for evaluation of metabolic disturbance in recently diagnosed rheumatoid arthritis patients. Rheumatol Int 33:2283-2289

28. Reaven GM (2008) Insulin resistance: the link between obesity and cardiovascular disease. Endocrinol Metab Clin North Am 37(3): 581-601, vii-viii

29. Elkan AC, Håkansson N, Frostegård J et al (2011) Low level of physical activity in women with rheumatoid arthritis is associated with cardiovascular risk factors but not with body fat mass-a cross sectional study. BMC Musculoskelet Disord 12:13

30. Sokka T, Hakkinen A, Kautiainen H et al (2008) Physical inactivity in patients with rheumatoid arthritis: data from twenty-one countries in a cross-sectional, international study. Arthritis Rheum 59: $42-50$

31. Hotamisligil GS (1999) Mechanisms of TNF-alpha-induced insulin resistance. Exp Clin Endocrinol Diabetes 107:119-125

32. Otero M, Lago R, Gomez R et al (2006) Changes in plasma levels of fat-derived hormones adiponectin, leptin, resistin and visfatin in patients with rheumatoid arthritis. Ann Rheum Dis 65:1198-1201

33. Bokarewa M, Nagaev I, Dahlberg L et al (2005) Resistin, an adipokine with potent proinflammatory properties. J Immunol 174:5789-5795

34. Hizmetli S, Kisa M, Gokalp N et al (2007) Are plasma and synovial fluid leptin levels correlated with disease activity in rheumatoid arthritis? Rheumatol Int 27:335-338

35. Laurberg TB, Frystyk J, Ellingsen T et al (2009) Plasma adiponectin in patients with active, early, and chronic rheumatoid arthritis who are steroid- and disease-modifying antirheumatic drug-naive compared with patients with osteoarthritis and controls. J Rheumatol 36: 1885-1891

36. Migita K, Maeda Y, Miyashita T et al (2006) The serum levels of resistin in rheumatoid arthritis patients. Clin Exp Rheumatol 24: 698-701

37. Yoshino T, Kusunoki N, Tanaka N et al (2011) Elevated serum levels of resistin, leptin, and adiponectin are associated with $\mathrm{C}$ reactive protein and also other clinical conditions in rheumatoid arthritis. Intern Med 50:269-275

38. Rho YH, Chung CP, Solus JF et al (2010) Adipocytokines, insulin resistance, and coronary atherosclerosis in rheumatoid arthritis. Arthritis Rheum 62:1259-1264

39. Toms TE, Panoulas VF, John H et al (2009) Methotrexate therapy associates with reduced prevalence of the metabolic syndrome in rheumatoid arthritis patients over the age of 60- more than just an anti-inflammatory effect? A cross sectional study. Arthritis Res Ther 11:R110

40. van Tuyl LH, Boers M, Lems WF et al (2010) Survival, comorbidities and joint damage 11 years after the COBRA combination 
therapy trial in early rheumatoid arthritis. Ann Rheum Dis 69:807812

41. Hoes JN, van der Goes MC, van Raalte DH et al (2011) Glucose tolerance, insulin sensitivity and beta-cell function in patients with rheumatoid arthritis treated with or without low-to-medium dose glucocorticoids. Ann Rheum Dis 70:1887-1894

42. Cansu B, Cansu DU, Kasifoglu T et al (2011) Disease-modifying antirheumatic drugs increase serum adiponectin levels in patients with rheumatoid arthritis. J Clin Rheumatol 17:14-17

43. Gonzalez-Gay MA, García-Unzueta MT, González-Juanatey C et al (2008) Anti-TNF-alpha therapy modulates resistin in patients with rheumatoid arthritis. Clin Exp Rheumatol 26:311-316
44. Popa C, Netea MG, de Graaf J et al (2009) Circulating leptin and adiponectina concentrations during tumor necrosis factor blockade in patients with active rheumatoid arthritis. J Rheumatol 36:724-730

45. Georgiadis AN, Papavasiliou EC, Lourida ES et al (2006) Atherogenic lipid profile is a feature characteristic of patients with early rheumatoid arthritis: effect of early treatment—a prospective, controlled study. Arthritis Res Ther 8:R82

46. Dessein PH, Stanwix AE, Joffe BI (2002) Cardiovascular risk in rheumatoid arthritis versus osteoarthritis: acute phase response related decreased insulin sensitivity and high-density lipoprotein cholesterol as well as clustering of metabolic syndrome features in rheumatoid arthritis. Arthritis Res 4:R5 